\title{
Ehrhart theory, modular flow reciprocity, and the Tutte polynomial
}

\author{
Felix Breuer • Raman Sanyal
}

Received: 8 March 2010 / Accepted: 17 August 2010 / Published online: 17 November 2010

(C) The Author(s) 2010. This article is published with open access at Springerlink.com

\begin{abstract}
Given an oriented graph $G$, the modular flow polynomial $\phi_{G}(k)$ counts the number of nowhere-zero $\mathbb{Z}_{k}$-flows of $G$. We give a description of the modular flow polynomial in terms of (open) Ehrhart polynomials of lattice polytopes. Using Ehrhart-Macdonald reciprocity we give a combinatorial interpretation for the values of $\phi_{G}$ at negative arguments which answers a question of Beck and Zaslavsky (Adv Math 205:134-162, 2006). Our construction extends to $\mathbb{Z}_{\ell}$-tensions and we recover Stanley's reciprocity theorem for the chromatic polynomial. Combining the combinatorial reciprocity statements for flows and tensions, we give an enumerative interpretation for positive evaluations of the Tutte polynomial $t_{G}(x, y)$ of $G$.
\end{abstract}

Keywords Nowhere-zero flows - Modular flow polynomial - Reciprocity theorems · Lattice-point counting $\cdot$ Ehrhart theory $\cdot$ Tutte polynomial

Mathematics Subject Classification (2000) Primary 05C31 Graph polynomials; Secondary 05C21 Flows in graphs · 52B20 Lattice polytopes · 52C35 Arrangements of points, flats, hyperplanes

Felix Breuer was supported by the Deutsche Forschungsgemeinschaft within the research training group 'Methods for Discrete Structures' (GRK 1408). Raman Sanyal was supported by the Konrad-Zuse-Zentrum für Informationstechnik Berlin and by a Miller Research Fellowship.

\section{F. Breuer}

Institut für Mathematik, Freie Universität Berlin, Arnimallee 3, 14195 Berlin, Germany

e-mail: felix.breuer@fu-berlin.de

R. Sanyal ( $\varangle)$

Department of Mathematics, University of California, Berkeley, 970 Evans Hall,

Berkeley, CA 94720, USA

e-mail: sanyal@math.berkeley.edu 


\section{Introduction}

The chromatic polynomial of a graph is probably the most famous graph polynomial. In 1973, Stanley [18] gave an "unorthodox" interpretation of graph colorings in terms of acyclic orientations and compatible maps. The benefit of this interpretation is a natural, combinatorial interpretation of (suitably normalized) evaluations of the chromatic polynomial at a negative argument. In some sense this was one of the first combinatorial reciprocity theorems [19]. In 2006, Beck and Zaslavsky [3] gave a different perspective on this result by casting it into the realms of geometry. They identified graph colorings as lattice points "inside" a polytope but "outside" a hyperplane arrangement—an object answering to the name of inside-out polytope. Thus, the chromatic polynomial can be understood as a sum of Ehrhart functions and a suitably generalized Ehrhart-Macdonald reciprocity yields the combinatorial interpretation. We explain more of the details in the sections to come. For a general background on Ehrhart polynomials and Ehrhart-Macdonald reciprocity we refer the reader to [2].

An equally important polynomial invariant of a graph is given by the modular flow polynomial. Let $G=(V, E)$ be an oriented graph and let $\mathcal{A}$ be an abelian group. An $\mathcal{A}$-flow is an assignment $f: E \rightarrow \mathcal{A}$ such that at every vertex we have a conservation of flow, i.e.,

$$
\sum_{u v \in E} f_{u v}-\sum_{v u \in E} f_{v u}=0
$$

for every $v \in V$. The support of the flow $f$ is $\operatorname{supp}(f)=\left\{e \in E: f_{e} \neq 0\right\}$ and $f$ is called nowhere-zero if $\operatorname{supp}(f)=E$. Tutte [20] was the first to consider nowhere-zero flows for a fixed group $\mathcal{A}$. He proved that the number of nowhere-zero $\mathcal{A}$-flows depends only on the order of the group and that $\phi_{G}(k)$, the number of nowhere-zero $\mathbb{Z}_{k}$-flows, is a polynomial in $k$. Clearly, this is only meaningful for finite groups, but in the case of $\mathbb{Z}$-flows a natural concept is that of a $k$-flow which is a $\mathbb{Z}$-flow with values strictly smaller than $k$ in absolute values. Tutte [21] proved that there is a nowhere-zero $\mathbb{Z}_{k}$-flow if and only if there is a nowhere-zero $k$-flow. However, the number of nowhere-zero $k$-flows and $\mathbb{Z}_{k}$-flows differ in general. Let us emphasize that the two flow polynomials are invariant under reorientations of $G$ and therefore are an invariant of the underlying unoriented graph of $G$. However, working with oriented graphs yields cleaner and more transparent results.

In 2002, Kochol [12] proved that $\bar{\phi}_{G}(k)$, the number of nowhere-zero $k$-flows, is also a polynomial and in [4] Beck and Zaslavsky showed that this is yet another incarnation of Ehrhart theory of inside-out polytopes. Moreover, this approach yields a reciprocity statement that parallels that for the chromatic polynomial: $(-1)^{\xi(G)} \bar{\phi}_{G}(-k)$ counts pairs of $k$-flows and compatible totally cyclic orientations. This raised the question for a combinatorial reciprocity theorem of the modular flow polynomial (cf. [4, Problem 3.2]).

As an answer to this question, the first result of this paper gives an interpretation of $(-1)^{\xi(G)} \phi_{G}(-k)$ as naturally counting pairs of $\mathbb{Z}_{k}$-flows and totally cyclic reorientations on certain subgraphs. We give the precise statement in Sect. 2. A little surprisingly, our proof is somewhat simpler than the one for $k$-flows in [4]. For starters, we do not need the theory of inside-out polytopes per se; in Sect. 3 we relate our proof to inside-out polytopes which sheds "geometric light" on some well-known properties of flow polynomials. In Sect. 4, we discuss colorings and their relations to $\mathbb{Z}_{k}$-tensions. We sketch how analogous arguments yield a reciprocity statement for $\mathbb{Z}_{k}$-tensions that corresponds to Stanley's reciprocity for colorings [18]. In Sect. 5 we make use of the reciprocity statements to prove a enumerative interpretation for arbitrary evaluations of Tutte polynomials of graphs at positive arguments, which is implicit in the work of Reiner [15]. In the appendices we give traditional, that is deletion-contraction, proofs for the main results of Sects. 2 and 5. 


\section{Modular flow reciprocity}

Let $G=(V, E)$ be an oriented graph, that is, an unoriented graph equipped with an orientation of its edges. We allow, even encourage, $G$ to have multiple edges and loops. For an $S \subseteq E$ we denote by $G_{\backslash S}, G_{/ S}$, and $G[S]$ the result of deleting, contracting, and restricting to $S$, respectively. Moreover, we denote by ${ }_{S} G$ the reorientation of $G$ along $S$, i.e., the graph obtained by reversing the orientation of the edges in $S$. We denote by $c(G)$ the number of (weakly) connected components and we call $e \in E$ a coloop or bridge if $c\left(G_{\backslash e}\right)=c(G)+1$. Finally, we denote by $\xi(G):=|E|-|V|+c(G)$ the cyclotomic number of $G$.

Let us give a precise definition for the main character.

Definition 2.1 For an oriented graph $G=(V, E)$, the modular flow polynomial $\phi_{G}$ of $G$ is the function

$$
\phi_{G}(k)=\#\left\{f: E \rightarrow \mathbb{Z}_{k}: f \text { nowhere-zero } \mathbb{Z}_{k} \text {-flow }\right\} .
$$

The name was justified by Tutte [21] who showed that $\phi_{G}$ is indeed a polynomial of degree $\xi(G)$. In particular, $\phi_{G}$ can be extended to negative arguments. In order to state our main result of this section we need the notion of a totally cyclic orientation. An oriented graph is called totally cyclic if every edge is contained in a directed cycle and $\sigma \subseteq E$ is a totally cyclic reorientation if ${ }_{\sigma} G$ is totally cyclic.

Theorem 2.2 (Modular flow reciprocity) Let $G=(V, E)$ be an oriented graph and let $k$ be a positive integer. Then $(-1)^{\xi(G)} \phi_{G}(-k)$ counts pairs $(f, \sigma)$ where $f$ is a $\mathbb{Z}_{k}$-flow and $\sigma \subseteq E \backslash \operatorname{supp}(f)$ is a totally cyclic reorientation for $G / \operatorname{supp}(f)$.

Let us remark that our result differs from the reciprocity theorem for $k$-flows (cf. [4, $\mathrm{Thm} 3.1 \mathrm{~b}]$ ) inasmuch that the flow and the reorientation are not subject to a compatibility constraint. Rather, we reorient at most those edges $e$ with $f(e)=0$ in the first place and contract all other edges.

Let us illustrate the result with two examples that will accompany us throughout.

Example 1 Consider the following graph $G_{1}$ with two vertices and three parallel and identically oriented edges $e_{1}, e_{2}, e_{3}$.

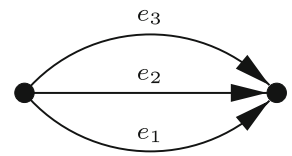

The flow conservation for a flow $f$ is given by $f\left(e_{1}\right)+f\left(e_{2}\right)+f\left(e_{3}\right)=0$ and this readily yields the number of nowhere-zero $\mathbb{Z}_{k}$-flows as $\phi_{G_{1}}(k)=(k-1)(k-2)$. The cyclotomic number of $G_{1}$ is $\xi\left(G_{1}\right)=2$ and hence $(-1)^{2} \phi_{G_{1}}(-k)=(k+1)(k+2)$. Now let us count the number of pairs $(f, \sigma)$ stated in Theorem 2.2 according to $n_{f}=|\operatorname{supp}(f)|$. For $n_{f}=0, f$ is the unique zero-flow and the number of totally cyclic orientations is 6 . The case $n_{f}=1$ does not show up and for $n_{f}=2$ there are exactly $\left(\begin{array}{l}3 \\ 2\end{array}\right)$ choices of non-zero edges and $(k-1)$ flows each time. The contraction in each case yields a loop which has two totally cyclic reorientations. Together with $n_{f}=3$, in which case we count the number of nowhere-zero $\mathbb{Z}_{k}$-flows, we get $(k-1)(k-2)+6(k-1)+6=(k+1)(k+2)$. 
Example 2 Our second example is the multigraph $G_{2}$ :

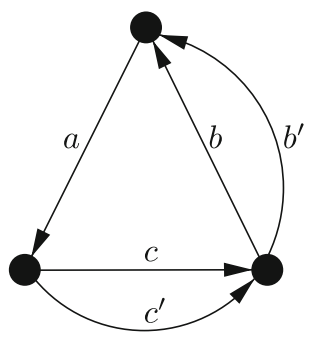

In order to compute the flow polynomial, consider the case of a 3-cycle $a, b, c$ without parallel edges. It is clear that the flow is determined by the (non-zero) value on the edge $a$ and the flow polynomial is thus $k-1$. Now every nowhere-zero flow on the 3 -cycle yields $(k-2)^{2}$ nowhere-zero flows on $G_{2}$ since flow on $b$ and $c$ can be "rerouted" through $b^{\prime}$ and $c^{\prime}$ as long as all remain nonzero. Hence, $\phi_{G_{2}}(k)=(k-1)(k-2)^{2}$ and $(-1)^{\xi\left(G_{2}\right)} \phi_{G_{2}}(-k)=(k+1)(k+2)^{2}$ with $\xi\left(G_{2}\right)=3$. The argument extends to counting the pairs $(f, \sigma)$ combinatorially, by lifting the pairs from the 3-cycle.

We will now set the stage for the proof of Theorem 2.2 which will mainly consist of casting the statement of Theorem 2.2 into a discrete geometric statement involving lattice polytopes. As a first step we will identify $\mathbb{Z}_{k}$ with a set of coset representatives given by the integers $0,1, \ldots, k-1$. With this identification the flow conservation at a vertex $v \in V$ can be rephrased as

$$
\begin{array}{cr}
\sum_{u v \in E} f_{u v}-\sum_{v u \in E} f_{v u}=0 & \text { over } \mathbb{Z}_{k} \\
\Leftrightarrow \sum_{u v \in E} f_{u v}-\sum_{v u \in E} f_{v u} \equiv 0 & \bmod k \\
\Leftrightarrow \sum_{u v \in E} f_{u v}-\sum_{v u \in E} f_{v u}=k \cdot b_{v} & \text { for some } b_{v} \in \mathbb{Z} .
\end{array}
$$

Letting $A=A_{G} \in\{0, \pm 1\}^{V \times E}$ be the incidence matrix of $G$, the last equivalence yields the following polyhedral reformulation: A point $f \in \mathbb{Z}^{E}$ represents a nowhere-zero $\mathbb{Z}_{k}$-flow if there is a $b \in \mathbb{Z}^{V}$ such that $f$ is contained in $\left(k \cdot P_{G}^{\circ}(b)\right) \cap \mathbb{Z}^{E}$ where

$$
P_{G}^{\circ}(b):=\left\{p \in \mathbb{R}^{E}: A p=b, 0<p_{e}<1 \text { for all } e \in E\right\} .
$$

Note that for such a $b, P_{G}^{\circ}(b)$ is a relatively open polytope of $\operatorname{dimension} \operatorname{dim} P_{G}^{\circ}(b)=$ rank $A_{G}=\xi(G)$. Denote by $\mathcal{B}_{G}=\left\{b \in \mathbb{Z}^{V}: P_{G}^{\circ}(b) \neq \emptyset\right\}$ the collection of all feasible $b$ 's. The set $\mathcal{B}_{G}$ is clearly finite (since the cube is compact) and for distinct $b, b^{\prime} \in \mathcal{B}_{G}$, the relatively open polytopes $P_{G}^{\circ}(b)$ and $P_{G}^{\circ}\left(b^{\prime}\right)$ are necessarily disjoint. The incidence matrix $A_{G}$ of an oriented graph is totally unimodular (cf., for example, [16, Sec. 19.3, Ex 2]). This remains true if we add rows that contain just a single 1 to encode constraints like $0 \leq p_{e} \leq 1$. Standard methods (cf. [16, Thm. 19.1]) then imply that the closure $P_{G}(b)=\overline{P_{G}^{\circ}(b)}$ is a vertex induced subpolytope of the $|E|$-dimensional standard cube. In particular, $P_{G}(b)$ has all its vertices in the standard lattice $\mathbb{Z}^{E}$, that is, $P_{G}(b)$ is a lattice polytope.

Example 1 (continued) The edge space of $G_{1}$ is three dimensional. The incidence matrix of $G_{1}$ is

$$
A=A_{G_{1}}=\left(\begin{array}{ccc}
-1 & -1 & -1 \\
1 & 1 & 1
\end{array}\right)
$$


It follows that $P_{G_{1}}^{\circ}(b)=\left\{x \in \mathbb{R}^{3}: 0<x_{1}, x_{2}, x_{3}<1, x_{1}+x_{2}+x_{3}=-b_{1}, x_{1}+x_{2}+x_{3}=b_{2}\right\}$ is non-empty iff $b^{\prime}=(-1,1)$ or $b^{\prime \prime}=(-2,2)$. The following figure shows the two polytopes as slices of the cube. The points correspond to the 6 nowhere-zero $\mathbb{Z}_{4}$-flows.

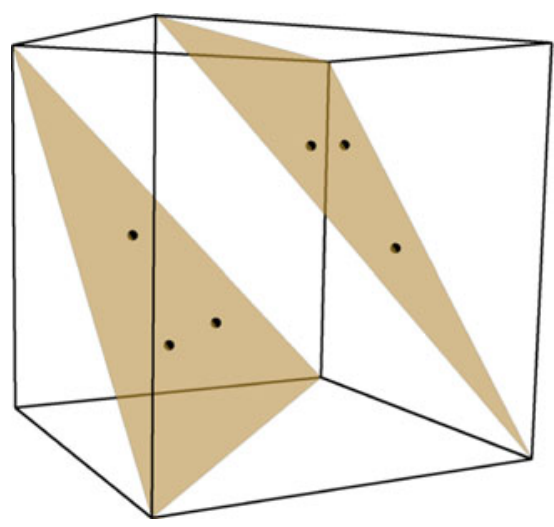

For a polytope $P \subset \mathbb{R}^{d}$ and $k \in \mathbb{Z}_{>0}$ let us denote by $\operatorname{Ehr}(P ; k)=\left|(k \cdot P) \cap \mathbb{Z}^{d}\right|$ the number of lattice points in the $k$-th dilate of $P$. Ehrhart [9] showed that if $P$ is a lattice polytope then $\operatorname{Ehr}(P ; k)$ is a polynomial in $k$ of degree $\operatorname{dim} P$.

Proposition 2.3 Let $G=(V, E)$ be an oriented graph. Then

$$
\phi_{G}(k)=\sum_{b \in \mathcal{B}_{G}} \operatorname{Ehr}\left(P_{G}^{\circ}(b) ; k\right)
$$

is a sum of Ehrhart polynomials.

Probably the most appealing feature of Ehrhart theory is that Ehrhart polynomials adhere to a beautiful geometric reciprocity that gives a meaning to the evaluation $\operatorname{Ehr}(P ;-k)$. For details see [2, Sect. 4].

Theorem 2.4 (Ehrhart-Macdonald reciprocity) Let $P$ be a lattice polytope and denote by $P^{\circ}$ the (relative) interior of $P$. Then

$$
\operatorname{Ehr}\left(P^{\circ} ; k\right)=(-1)^{\operatorname{dim} P} \operatorname{Ehr}(P ;-k) .
$$

In light of Proposition 2.3 together with Ehrhart-Macdonald reciprocity it is sufficient to give a combinatorial meaning to the lattice points in the boundary of $k \cdot P_{G}(b)$. Fix a $b \in \mathcal{B}_{G}$ and let $F \subset P_{G}(b)$ be a proper face. As $P_{G}(b)$ is a section of the $|E|$-cube, there is a partition $\sigma_{-} \cup \sigma_{0} \cup \sigma_{+}=E$ into disjoint parts such that the relative interior of $F$ is given by all the points $p \in P_{G}(b)$ such that

$$
\begin{aligned}
0=p_{e} \quad & \text { for } e \in \sigma_{-}, \\
0<p_{e}<1 & \text { for } e \in \sigma_{0}, \text { and } \\
& p_{e}=1 \text { for } e \in \sigma_{+} .
\end{aligned}
$$

A lattice point $f$ in the relative interior of $k \cdot F$ represents a $\mathbb{Z}_{k}$-flow but, since $0 \equiv k \bmod k$, this representation is not unique. However, if $f^{\prime} \in\left(k \cdot P_{G}\left(b^{\prime}\right)\right) \cap \mathbb{Z}^{E}$ for some $b^{\prime} \in \mathcal{B}_{G}$ yields the same $\mathbb{Z}_{k}$-flow modulo $k$ but is different from $f$ otherwise, then $f^{\prime}$ is in the relative 
interior of $k \cdot F^{\prime}$ where $F^{\prime} \subset P_{G}\left(b^{\prime}\right)$ is a proper face and $F \neq F^{\prime}$. Thus, the idea is to keep track of the origin of $f$. This leads to reorientations on the contraction.

To this end, let $z_{F}$ and $z_{P}$ be points in the relative interiors of $F$ and $P_{G}(b)$ respectively and consider $z:=z_{P}-z_{F}$. Then $z \in \operatorname{ker} A_{G}$ and we can predict the sign of $z_{e}$ for $e \in \sigma_{-} \cup \sigma_{+}$. The next lemma relates the kernel of $A_{G}$ to totally cyclic reorientations of $G$.

Lemma 2.5 [11, Lem. 8.1] Let $G=(V, E)$ be a graph and $A=A_{G}$ its incidence matrix. The connected components of $\operatorname{ker} A \backslash\left\{p \in \mathbb{R}^{E}: p_{e}=0\right.$ for some $\left.e \in E\right\}$ are in bijection with the totally cyclic reorientations of $G$. The totally cyclic reorientation $\sigma$ associated to $a$ connected component is $\sigma=\left\{e \in E: p_{e}<0\right\}$ for an arbitrary point $p$ in that component.

The restriction $\tilde{z}$ of $z$ to $\sigma_{+} \cup \sigma_{-}$is an element of ker $A_{G / \sigma_{0}}$ and Lemma 2.5 then asserts that $\sigma_{+}$is a totally cyclic reorientation for $G_{/ \sigma_{0}}$ and uniquely identifies the face $F$ for which $z_{F}$ is in the relative interior.

Proof of Theorem 2.2 Let $f \in k \cdot[0,1]^{E} \cap \mathbb{Z}^{E}$ be a lattice point in the $k$-th dilate of the $|E|$-dimensional standard cube. Let $\sigma(f)=\left\{e \in E: f_{e}=k\right\}$ and denote by $\bar{f}$ the point $f$ modulo $k$ componentwise.

By our discussion and, in particular, Ehrhart-Macdonald reciprocity we have that

$$
(-1)^{\xi(G)} \phi_{G}(-k)=\left|\bigcup_{b \in \mathcal{B}_{G}}\left(k \cdot P_{G}(b)\right) \cap \mathbb{Z}^{E}\right|
$$

where the union on the right-hand side is over disjoint sets. The theorem follows by proving that $f \mapsto(\bar{f}, \sigma(f))$ is a bijection between points in the right-hand side and pairs of $\mathbb{Z}_{k}$-flows and totally cyclic orientations on the contraction of the support. However, by our previous reasoning it is clear that this is a well-defined map and we are left with showing that there exists an inverse mapping.

Let $(\bar{f}, \sigma)$ be a pair with $\bar{f}: E \rightarrow \mathbb{Z}_{k}$ a $\mathbb{Z}_{k}$-flow and $\sigma \subseteq E \backslash \operatorname{supp}(\bar{f})$ a totally cyclic reorientation of $G_{/ \operatorname{supp}(\bar{f})}$. Let $f^{\prime} \in k \cdot[0,1]^{E} \cap \mathbb{Z}^{E}$ be the unique point with $f_{e}^{\prime}=k$ iff $e \in \sigma$ and $\overline{f^{\prime}}=\bar{f}$. The point $f^{\prime}$ is in the boundary of $P_{G}(b)$ for $b=A f^{\prime}$ and we are done if we can show that $b \in \mathcal{B}_{G}$. Now, by Lemma 2.5 , we can pick a vector $z \in \mathbb{R}^{E}$ with $A z=0, z_{e}<0$ for $e \in \sigma$ and $z_{e}>0$ for $e \notin \operatorname{supp}(\bar{f}) \cup \sigma$. Thus, for $\varepsilon>0$ sufficiently small, $f^{\prime}+\varepsilon z$ is a point of $P_{G}(b)$ in the interior of the cube and this concludes the argument.

As an immediate Corollary we get the following known enumerative result.

Corollary 2.6 [18, Cor. 1.3] Let $G=(V, E)$ be an oriented graph. Then $(-1)^{\xi(G)} \phi_{G}(-1)$ is the number of totally cyclic reorientations of $G$.

In particular, every totally cyclic reorientation belongs to exactly one $P_{G}(b)$. It is worthwhile interpreting this partition of totally cyclic reorientations as an equivalence relation. The following proposition phrases the equivalence in combinatorial terms. For a set $\sigma \subseteq E$ we denote by $e_{\sigma} \in\{0,1\}^{E}$ the characteristic vector of $\sigma$.

Proposition 2.7 Let $G=(V, E)$ be an oriented graph and $\sigma, \sigma^{\prime} \subseteq E$ two totally cyclic reorientations. The points $e_{\sigma}, e_{\sigma^{\prime}} \in\{0,1\}^{E}$ are both vertices of $P_{G}(b)$ for some $b \in \mathcal{B}_{G}$ if and only if $\sigma_{\sigma} G$ can be obtained from $\sigma_{\sigma^{\prime}} G$ by the reversal of directed cycles.

Proof The points $e_{\sigma}, e_{\sigma^{\prime}} \in\{0,1\}^{E}$ are both contained in a common $P_{G}(b)$ iff $z:=e_{\sigma}-e_{\sigma^{\prime}}$ is an element of $\operatorname{ker} A_{G}$. By [11, Lem. 8.5], $z$ is a linear combination with non-negative coefficients of orientations of cycles of $G$. 
For an oriented graph $G=(V, E)$, we denote by $I_{G} \in \mathbb{Z}^{V}$ the in-degree sequence, that is the number $\left(I_{G}\right)_{v}$ of incoming edges for every vertex $v \in V$. Similar, we define the out-degree sequence $O_{G} \in \mathbb{Z}^{V}$ of $G$.

Theorem 2.8 Let $G=(V, E)$ be an oriented graph. Then $\mathcal{B}_{G}$ is in bijection with

$$
\left\{I_{\sigma} G: \sigma \subseteq E \text { totally cyclic reorientation }\right\} .
$$

Proof Let $A_{G}$ be the incidence matrix of $G$. It is clear that we can recover $I_{G}$ from the knowledge of the (undirected) degree sequence $D=I_{G}+O_{G}$ and $I_{G}-O_{G}=A_{G} \mathbb{1}=: b_{0}$. Now, for any reorientation $\sigma \subseteq E$, we have

$$
I_{\sigma}-O_{\sigma}=A_{\sigma} \mathbb{1}=A_{G}\left(\mathbb{1}-2 e_{\sigma}\right)=b_{0}-2 A_{G} e_{\sigma} .
$$

Hence, the in-degree sequence of ${ }_{\sigma} G$ is uniquely determined by $b_{\sigma}=A_{G} e_{\sigma}$. Moreover, the reversal of a directed cycle in $G$ leaves the in- and out-degree sequences invariant and thus is invariant within $P_{G}(b)$. Using that $\mathcal{B}_{G}=\left\{b=A_{G} e_{\sigma}: \sigma \subseteq E\right.$ totally cyclic reorientation $\}$ finishes the proof.

As $\phi_{G}$ is the sum of the (open) Ehrhart polynomials for $P_{G}^{\circ}(b)$, we recover the following result by Gioan [10]. It follows from the fact that constant term of the Ehrhart polynomial $\operatorname{Ehr}(P ; k)$ equals 1 (= the Euler characteristic of $P$ ).

Corollary 2.9 [10, Thm. 3.1] Let $G=(V, E)$ be an oriented graph and $t_{G}(x, y)$ its Tutte polynomial. Then

$$
t_{G}(0,1)=(-1)^{\xi(G)} \phi_{G}(0)=\sum_{b \in \mathcal{B}_{G}} \operatorname{Ehr}\left(P_{G}(b) ; 0\right)=\left|\mathcal{B}_{G}\right|
$$

is the number of in-degree sequences of totally cyclic reorientations of $G$.

The evaluation $t_{G}(0,1)$ has several known interpretations as, for example, the number of spanning trees with zero external activity, the Euler characteristic of the independence complex of the matroid $M_{G}$ associated to $G$, or the number of facets of the broken circuit complex of the dual matroid $M_{G}^{\perp}$. We refer the reader to the survey article by Brylawski and Oxley [6] for further details.

\section{Modular flows inside-out}

In this section we relate our previous construction to inside-out polytopes. The benefit in doing so will be a simple geometric explanation for the following fact.

Corollary 3.1 The modular flow polynomial of an oriented graph $G$ has degree $\xi(G)$ and leading coefficient 1.

This, in turn, is a consequence of the fact that the number of modular $\mathbb{Z}_{k}$-flows is a generalized Tutte-Grothendieck invariant (cf. Sect. 5) and, hence, obeys the following deletioncontraction property.

Proposition 3.2 ([6, Prop. 6.3.4]) Let $G=(V, E)$ be an oriented graph and $e \in E$ an edge. If e is neither a loop nor a coloop, then

$$
\phi_{G}(k)=\phi_{G / e}(k)-\phi_{G_{\backslash e}}(k) .
$$

Otherwise, $\phi_{G}(k)=(k-1) \phi_{G_{\backslash e}}(k)$ if e is a loop and $\phi_{G}(k)=0$ if e is a coloop. 
While the degree of the polynomial is clear from our interpretation in terms of Ehrhart polynomial, the fact that the leading coefficient is 1 is not.

Proposition 3.3 ([2, Cor. 3.20]) Let $P$ be a d-dimensional lattice polytope then the leading coefficient of $\operatorname{Ehr}(P ; k)$ is the volume $\operatorname{vol}(P)$ of $P$.

So, the best we can say so far is that

$$
1=\sum_{b \in \mathcal{B}_{G}} \operatorname{vol}\left(P_{G}(b)\right) .
$$

However, the answer we are aiming at is that suitably arranging the polytopes $P_{G}(b)$ yields a subdivision of a standard cube of dimension $\xi(G)$. Thus, the total volume of the $P_{G}(b)$ 's is that of the standard cube.

In particular, the subdivision of the cube is induced by an arrangement of hyperplanes and therefore directly leads to inside-out polytopes. For our needs an inside-out polytope is a pair $(P, \mathcal{H})$ consisting of a $d$-dimensional lattice polytope $P \subset \mathbb{R}^{d}$ and a hyperplane arrangement $\mathcal{H}=\left\{H_{1}, \ldots, H_{m}\right\}$ such that any flat that meets $P$ also meets the interior of $P$. The hyperplane arrangement is allowed to be infinite as long as only finitely many hyperplanes meet $P$. The open Ehrhart function of $(P, \mathcal{H})$ is the function $\operatorname{Ehr}\left(P^{\circ}, \mathcal{H} ; k\right)=\left|k \cdot\left(P^{\circ} \backslash \cup \mathcal{H}\right) \cap \mathbb{Z}^{d}\right|$. In the case of a lattice inside-out polytope, i.e., $\mathcal{H}$ subdivides $P$ into lattice polytopes, the open (as well as the closed) Ehrhart function is a polynomial of degree $d$ and leading coefficient $\operatorname{vol}(P)$.

Let $G=(V, E)$ be an oriented graph and let $T \subseteq E$ be a spanning forest, i.e., a spanning tree per component. Let $T^{c}=E \backslash T$ denote the edges not in the forest. We will use $T$ to construct a cycle basis, i.e., a basis for ker $A_{G}$, combinatorially. Let $C \in\{0, \pm 1\}^{T^{c} \times E}$ be the matrix with rows $C_{f}$ for $f \in T^{c}$ defined as follows: The support of $C_{f}$ • is given by the edges in the unique undirected cycle $K$ in $G[T \cup f]$. There is a unique reorientation of $K$ that makes it an oriented cycle and that fixes the orientation on $f$. The signs keep track of which edges have to be reoriented. It is known (see [17, Thm. 11.1]) that every flow is an integral linear combination of the $C_{f}$. This implies that every nowhere-zero $\mathbb{Z}_{k}$-flow corresponds to a unique point $h \in \mathbb{Z}^{T^{c}}$ with $0<h_{f}<k$ for $f \in T^{c}$ and $(h C)_{e} \not \equiv 0 \bmod k$ for all $e \in T$. Denote by $C_{\bullet}$ the columns of $C$ for $e \in T$, then this yields the following interpretation in terms of inside-out polytopes.

Proposition 3.4 Let $P=[0,1]^{T^{c}}$ be the standard cube in $\mathbb{R}^{T^{c}}$ and let $\mathcal{H}$ be the arrangement of hyperplanes $H_{e, d_{e}}=\left\{q \in \mathbb{R}^{T^{c}}: C_{\bullet e}^{T} q=d_{e}\right\}$ for $e \in T$ and $d_{e} \in \mathbb{Z}$. Then

$$
\phi_{G}(k)=\operatorname{Ehr}\left(P^{\circ}, \mathcal{H} ; k\right) .
$$

In some sense the above construction is dual to that presented in Sect. 2: whereas the linear conditions in the construction of Sect. 2 forced the points to be $\mathbb{Z}_{k}$-flows, the construction here satisfies that automatically, and violating the linear conditions enforces the nowhere-zero condition. This is an instance of Gale duality (cf. [14, Sect. 5.6]) applied to the hyperplane arrangement $\mathcal{H}$.

Example 1 (continued) For the choice of the spanning tree $T=\left\{e_{1}\right\}$, the cycle basis is

$$
C={ }_{e_{2}}^{e_{2}}\left(\begin{array}{lll}
e_{1} & e_{2} & e_{3} \\
-1 & 1 & 0 \\
-1 & 0 & 1
\end{array}\right) .
$$


The polytope $P$ is a 2-cube and the hyperplane arrangement $\mathcal{H}$ is given by $H_{e_{1}, d}=$ $\left\{\left(q_{1}, q_{2}\right) \in \mathbb{R}^{T^{c}}: q_{1}+q_{2}=-d\right\}$ for $d \in \mathbb{Z}$. The resulting inside-out polytope is the following.

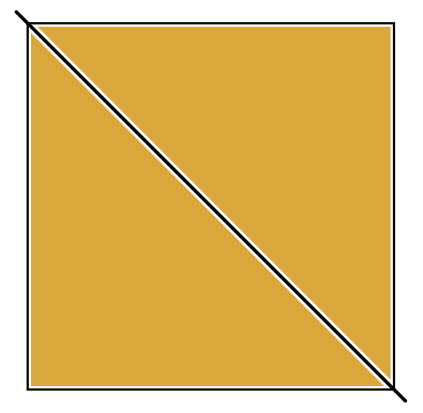

Example 2 (continued) For $G_{2}$ we pick the spanning tree $T=\{a, b\}$ and, hence, the cycle basis

$$
C={ }_{c}{ }_{c^{\prime}}\left(\begin{array}{rrrrr}
a & b & b^{\prime} & c & c^{\prime} \\
& -1 & 1 & & \\
1 & 1 & & 1 & \\
1 & 1 & & & 1
\end{array}\right) .
$$

So the two parallel classes of hyperplanes correspond to $H_{a, d_{a}}=\left\{\left(b^{\prime}, c, c^{\prime}\right) \in \mathbb{R}^{T^{c}}\right.$ : $\left.c+c^{\prime}=d_{a}\right\}$ and $H_{b, d_{b}}=\left\{\left(b^{\prime}, c, c^{\prime}\right) \in \mathbb{R}^{T^{c}}:-b^{\prime}+c+c^{\prime}=d_{b}\right\}$. The resulting inside-out polytope is:

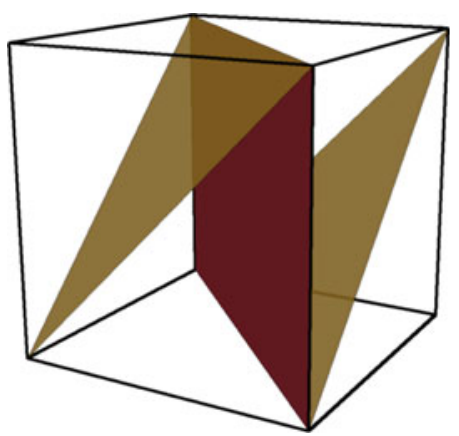

The four chambers correspond to the feasible $b$ 's.

So the leading coefficient of $\phi_{G}$ refers to the volume of a $\xi(G)$-dimensional standard cube $P=[0,1]^{T^{c}}$. The chambers, i.e., connected components of $P^{\circ} \backslash \cup \mathcal{H}$ are in bijection with $\mathcal{B}_{G}$. Indeed, every chamber is isomorphic to a unique $P_{G}^{\circ}(b)$ under the restriction to the coordinates $T^{c}$. With some more work it is possible to see the reciprocity in this picture. This line of thought has been pursued by Babson and Beck [1] via toric arrangements and by Chen and Stanley [8] via group arrangements.

One point worth mentioning is that the deletion-contraction property of the flow polynomial can be nicely observed in terms of the freedom of choice for a spanning forest. The contraction of an edge $e$ simply removes the family of hyperplanes $H_{e, d_{e}}$ from the arrangement. The resulting over count can be compensated for by subtracting the number of lattice 
points in the inside-out polytope obtained by restricting to this family. However, choosing a spanning forest not containing $e$ yields an inside-out polytope where the family $H_{e, d_{e}}$ corresponds to two parallel facets of the cube and restricting yields the inside-out polytope for $G_{\backslash e}$.

\section{Modular tensions and Stanley's reciprocity theorem}

In the introduction we mentioned Stanley's reciprocity theorem for the chromatic polynomial $\chi_{G}$ of a graph $G=(V, E)$. It turns out that this reciprocity is best observed in the related setting of modular tensions. In this section we sketch the changes to our previous constructions in order to accommodate modular tensions and we formulate a reciprocity theorem.

An $\ell$-coloring is a map $c: V \rightarrow\{0, \ldots, \ell-1\}$. An $\ell$-coloring $c$ is called proper if $c(u) \neq c(v)$ whenever $u$ and $v$ are adjacent in $G$ and the chromatic polynomial $\chi_{G}(\ell)$ counts the number of proper $\ell$-colorings of $G$. A reorientation $\sigma$ of $G$ is acyclic if no edge of $G$ lies on a directed cycle. A coloring $c$ and an acyclic reorientation $\sigma$ are compatible if for every edge $u v$ of ${ }_{\sigma} G$ we have $c(u) \leq c(v)$.

Theorem 4.1 (Stanley [18]) For an oriented graph $G=(V, E)$ and $\ell>0$ an integer, $(-1)^{|V|} \chi_{G}(-\ell)$ is the number of tuples $(c, \sigma)$ where $c$ is an $\ell$-coloring and $\sigma \subseteq E$ is a compatible acyclic reorientation.

The idea that leads to the notion of tensions is the following. Let us suppose for a moment that $G$ is connected. Then we can recover the coloring $c$ by knowing the initial color $c\left(v_{0}\right)$ of some vertex $v_{0}$ and the difference of the colors $t(u v):=c(u)-c(v)$ on each (oriented) edge $u v$. For this to make sense, we identify the set of colors with the group $\mathbb{Z}_{l}$ in which the difference of two colors makes sense. Thus, we recover the color on a vertex $w$ by adding and subtracting the tensions $t(e)$ of edges along a path from $v_{0}$ to $w$. Note that the color of $c(w)$ is independent of the chosen path and we take this as the defining property of tensions.

For a cycle $C \subseteq E$ in the underlying undirected graph, we denote by $C_{-} \subset C$ a collection of edges whose reorientation turns $C$ into a directed cycle and we let $C_{+}:=C \backslash C_{-}$be the remaining edges. Let $\mathcal{A}$ be an abelian group. A map $t: E \rightarrow \mathcal{A}$ is called an $\mathcal{A}$-tension if

$$
\langle C, t\rangle:=\sum_{e \in C_{+}} t(e)-\sum_{e \in C_{-}} t(e)=0
$$

for each undirected cycle $C \subseteq E$. Note that this is independent of the choice of $C_{-}$. An induced coloring $c$ is proper if and only if $t$ is nowhere-zero, that is $\operatorname{supp}(t):=\{e \in E$ : $t(e) \neq 0\}=E$. It can be shown that, as in the case of flows, the number of nowherezero $\mathcal{A}$-tensions depends only on the order of $\mathcal{A}$ and we define $\theta_{G}(\ell)$ to be the number of nowhere-zero $\mathbb{Z}_{\ell}$-tensions of $G$.

Every nowhere-zero $\mathbb{Z}_{\ell}$-tension $t$ yields $\left|\mathbb{Z}_{\ell}\right|^{c(G)}$ different colorings by choosing an initial color for every component and this proves the following known fact that $\chi_{G}(\ell)=\ell^{c(G)} \theta_{G}(\ell)$, i.e., the tension polynomial is a non-trivial factor of the chromatic polynomial. Hence, we arrive at the following equivalent reformulation of Theorem 4.1 which is already implicit in Stanley's work; see also Chen [7].

Theorem 4.2 Let $G=(V, E)$ be an oriented graph. Then $(-1)^{|V|-c(G)} \theta_{G}(-\ell)$ counts pairs $(t, \sigma)$ where $t$ is a $\mathbb{Z}_{\ell}$-tension on $G$ and $\sigma$ is an acyclic reorientation of $G \backslash \operatorname{supp}(t)$.

Equivalence of Theorems 4.1 and 4.2 In light of the fact that $\chi_{G}(\ell)=\ell^{c(G)} \theta_{G}(\ell)$ it suffices to argue that for a given $k$-coloring $c$ and a corresponding $\mathbb{Z}_{k}$-tension $t$, the acyclic reorientations of $G_{\backslash \operatorname{supp}(t)}$ are in bijection with the acyclic reorientations of $G$ that are compatible 
with $c$. If $\sigma$ is an acyclic reorientation of $G$ then clearly $\sigma \cap E \backslash \operatorname{supp}(t)$ is an acyclic reorientation of $G_{\backslash \operatorname{supp}(t)}$. Conversely, let $\sigma^{\prime}$ be an acyclic reorientation of $G_{\backslash \operatorname{supp}(t)}$. We have to show that there is a unique extension of $\sigma^{\prime}$ to an acyclic reorientation $\sigma$ of $G$ that is compatible with $c$. However, the condition that $e=u v$ has to be oriented from $u$ to $v$ whenever $c(u)<c(v)$ fixes the reorientation of all edges in $\operatorname{supp}(t)$. Suppose the resulting reorientation $\sigma$ did contain a directed cycle $C$. Then all vertices on $C$ have to have the same color with respect to $c$, as following an edge can never decrease the color. But this means that $C \subseteq E \backslash \operatorname{supp}(t)$ which is a contradiction to $\sigma^{\prime} G \backslash \operatorname{supp(t)}$ being acyclic.

Note the similarity to the statement of Theorem 2.2. This is not at all surprising from the (oriented) matroid point of view as we have the following correspondences:

$$
\begin{aligned}
\text { flow } f & \leftrightarrow \text { tension } t \\
\xi(G) & \leftrightarrow|V|-c(G) \\
\text { totally cyclic } & \leftrightarrow \operatorname{acyclic} \\
G_{/ \operatorname{supp}(f)} & \leftrightarrow G_{\backslash \operatorname{supp}(t)}
\end{aligned}
$$

Let us quickly remark on the conception of a "tension". If $\mathcal{A}$ is an ordered group, such as $\mathbb{Z}$, then the elements in $\mathcal{A}$ can be thought of heights and a tension measures the difference in altitude along an edge. In particular, if we reorient $G$ such that $t(e)>0$ for all $e$, then it is clear that every nowhere-zero $\mathbb{Z}$-tension yields an acyclic reorientation.

We refrain from giving a proof of Theorem 4.2 but instead supply the necessary modifications to the proofs for flow reciprocity in Sects. 2 and 3.

For an arbitrary spanning tree $T$, let $C$ be the cycle basis constructed in Sect. 3. It suffices to verify the defining properties of tensions on such a cycle basis as every cycle is a superposition of these elementary cycles. Thus, identifying $\mathbb{Z}_{\ell}=\{0, \ldots, \ell-1\}$, we have that $t: E \rightarrow \mathbb{Z}$ represents a nowhere-zero $\mathbb{Z}_{\ell}$-tension if $0<t(e)<\ell$ for every $e \in E$ and there is a $d \in \mathbb{Z}^{T^{c}}$ such that $C t=\ell \cdot d$. Analogously to the flow case, this formulation furnishes a collection of relatively open disjoint polytopes whose Ehrhart polynomials yield the nowhere-zero tension polynomial.

For the reciprocity the key lemma that yields the interpretation in terms of acyclic reorientations is

Lemma 4.3 ([11, Lem. 7.1]) Let $G=(V, E)$ be an oriented graph and $C$ a cycle basis. Then the connected components of $\operatorname{ker} C \backslash\left\{p \in \mathbb{R}^{E}: p_{e}=0\right.$ for some $\left.e \in E\right\}$ are in bijection with the acyclic reorientations of $G$.

Tensions can be parametrized by the edges in a spanning forest, leaving it to the non-forest edges $T^{c}$ to compensate along each cycle in the cycle basis $C$. This yields the description of an inside-out polytope analogous to the flow case in Sect. 3.

\section{An interpretation of the Tutte polynomial}

An (integral) generalized Tutte-Grothendieck (T-G) invariant is an assignment $f_{G} \in \mathbb{Z}$ to every graph $G$ such that for some constants $\tau_{f}, \sigma_{f} \in \mathbb{Z}$

(1) $f_{G}=\sigma_{f} f_{G_{\backslash e}}+\tau_{f} f_{G_{/ e}}$ for every $e \in E$ that is neither a loop nor a coloop, and

(2) $f_{G}=f_{G[e]} \cdot f_{G_{\backslash e}}$ otherwise.

A large collection of important invariants for graphs (or more generally matroids) qualifies as Tutte-Grothendieck invariants (cf. [6]), among them all evaluations of chromatic and modular flow and tension polynomials. The Tutte polynomial $t_{G}(x, y) \in \mathbb{Z}[x, y]$ of a graph $G$ is 
the unique function such that every generalized $\mathrm{T}-\mathrm{G}$ invariant $f_{G}$ can be expressed as

$$
f_{G}=\sigma_{f}^{\xi(G)} \tau_{f}^{|V|-c(G)} t_{G}\left(\frac{f_{I}}{\tau_{f}}, \frac{f_{L}}{\sigma_{f}}\right)
$$

where $f_{L}$ and $f_{I}$ is the invariant of a loop and coloop, respectively.

Insofar the Tutte polynomial expresses a multitude of enumerative invariants, but, to the best of our knowledge, there is no enumerative interpretation for arbitrary evaluations of the Tutte polynomial. The reciprocity statements of Sects. 2 and 4 yield a natural interpretation of what the Tutte polynomial evaluated at $(1+\ell, 1+k)$ for positive $k, \ell$ counts.

Theorem 5.1 Let $G$ be a graph and $k, \ell$ two positive integers. Then $t_{G}(1+\ell, 1+k)$ counts triples $(f, t, \sigma)$ where

(i) $\quad f$ is a $\mathbb{Z}_{k}$-flow and $t$ is a $\mathbb{Z}_{\ell}$-tension on $G$,

(ii) $f$ and $t$ have disjoint support, and

(iii) $\sigma \subseteq E \backslash \operatorname{supp}(f) \cup \operatorname{supp}(t)$ is a reorientation of $G \backslash \operatorname{supp}(f) \cup \operatorname{supp}(t)$.

The polynomial $t_{G}(1+\ell, 1+k)$ is also known as the rank polynomial of $G$.

In Appendix B, we give a proof of Theorem 5.1 from first principles, i.e., we prove that the stated cardinality itself is a generalized T-G invariant with structure constants $\tau=\sigma=1$ and values $1+k$ and $1+\ell$ for loops and coloops, respectively. Here, however, we give a proof by noting that Theorem 5.1 is equivalent to the convolution formula for Tutte polynomials of Kook et al. [13] specialized to graphs.

We need the following observation regarding reorientations of graphs.

Lemma 5.2 Let $G=(V, E)$ be an oriented graph. Then there is a unique $S \subseteq E$ such that $G[S]$ is totally cyclic and $G_{/ S}$ is acyclic.

Proof Let $S \subseteq E$ be the collection of edges that lie on a directed cycle in $G$. Then, clearly, $G[S]$ is totally cyclic and, as $G[S]$ is componentwise strongly connected, $G_{/ S}$ is acyclic. As for uniqueness, suppose that $S^{\prime} \subseteq E$ has the same properties. As $G\left[S^{\prime}\right]$ is totally cyclic, clearly $S^{\prime} \subset S$. Now if $e \in S \backslash S^{\prime}$, then $e$ is contained in a directed cycle which remains true in $G_{/ S^{\prime}}$, in contradiction to $G_{/ S^{\prime}}$ being acyclic. Hence $S=S^{\prime}$.

Proof of Theorem 5.1 Consider the collection of triples $(f, t, \sigma)$ as in the theorem. For every triple, we claim that there is a unique $S \subseteq E$ such that

(1) $f$ is a $\mathbb{Z}_{k}$-flow on $G[S]$ and $\sigma \cap S$ is a totally cyclic reorientation of $(G[S]) / \operatorname{supp(f)}$, and

(2) $t$ is a $\mathbb{Z}_{\ell}$-tension on $G_{/ S}$ such that $\sigma \backslash S$ is an acyclic reorientation of $\left(G_{/ S}\right) \backslash \operatorname{supp}(t)$.

Indeed, let $S^{\prime} \subseteq E$ be the set for ${ }_{\sigma} G / \operatorname{supp}(f) \backslash \operatorname{supp}(t)$ whose existence and uniqueness is asserted by Lemma 5.2. Now, it is easy to verify that $S:=S^{\prime} \cup \operatorname{supp}(f)$ is the unique set with the properties above.

In light of Theorems 2.2 and 4.2, the number of triples for which $S$ is the unique set obeying the above properties is

$$
(-1)^{\xi(G[S])} \phi_{G[S]}(-k) \cdot(-1)^{|V|-c\left(G_{/ S}\right)} \theta_{G_{/ S}}(-\ell)
$$

The flow and tension polynomials are both specializations of the Tutte polynomial and hence

$$
\begin{aligned}
t_{G[S]}(0,1+k) & =(-1)^{\xi(G[S])} \phi_{G[S]}(-k) \\
t_{G / S}(1+\ell, 0) & =(-1)^{|V|-c\left(G_{/ S}\right)} \theta_{G_{/ S}}(-\ell) .
\end{aligned}
$$


To finish the proof, we recall the result of Kook et al. [13, Thm. 1] stating that

$$
t_{G}(1+\ell, 1+k)=\sum_{S \subseteq E} t_{G[S]}(0,1+k) t_{G_{/ S}}(1+\ell, 0) .
$$

This interpretation yields the following counting formula which matches a result of Reiner [15] but removes the restriction to prime powers. It follows from Theorem 5.1 together with the fact that every flow or tension is nowhere zero restricted to its support.

Corollary 5.3 Let $G=(V, E)$ be a graph, then the Tutte polynomial is given by

$$
t_{G}(1+\ell, 1+k)=\sum_{S \subseteq T \subseteq E} 2^{|T \backslash S|} \phi_{G[S]}(k) \theta_{G_{/ T}}(\ell)
$$

Let us remark that enumerative interpretations for all evaluations of the Tutte polynomial are not to be expected as for negative parameters the sign of $t_{G}(x, y)$ depends on the magnitude of the arguments. However, our interpretation misses some fundamental evaluations such as $t_{G}(1,2)$ for the number of spanning sets and $t_{G}(2,1)$ for the number of independent sets. We also remark that interpretations for evaluations of the flow- and tension polynomials at negative values also yield interpretations in the spirit of [15, Cor. 2] for the missing two orthants $(1-\ell, 1+k)$ and $(1+\ell, 1-k)$. In this way, the reciprocity theorems for the modular flow and tension polynomials can be combined with the convolution formula of Kook et al. to yield a unified framework in which the value of the Tutte polynomial at every lattice point in the plane can be interpreted. This is worked out in [5, Sect. 3.11].

Acknowledgments We would like to thank Matthias Beck for valuable conversations and comments on an earlier version of this paper.

Open Access This article is distributed under the terms of the Creative Commons Attribution Noncommercial License which permits any noncommercial use, distribution, and reproduction in any medium, provided the original author(s) and source are credited.

\section{Appendix A: Combinatorial proof of modular flow reciprocity}

In this section we give a combinatorial proof of Theorem 2.2. Our approach is straightforward: we show that $(-1)^{\xi(G)} \phi_{G}(-k)$ is a generalized Tutte-Grothendieck invariant with the correct structure constants.

Let $\mathrm{F}_{G}(k)$ denote the set of all pairs $(f, \sigma)$ of a $\mathbb{Z}_{k}$-flow $f$ and a totally cyclic reorientation $\sigma$ of $G_{/ \operatorname{supp}(f)}$. Using this notation Theorem 2.2 simply states

$$
(-1)^{\xi(G)} \phi_{G}(-k)=\#_{G}(k) .
$$

In light of Proposition 3.2 it suffices to show that $\mathrm{AF}_{G}(k)$ is a Tutte-Grothendieck invariant with the structure constants as given in the following theorem.

Theorem A.1 Let $G=(V, E)$ be an oriented graph and let $k \in \mathbb{N}$.

(1) If $E=\emptyset$, then $\# \mathrm{~F}_{G}(k)=1$.

(2) If $e \in E$ is a coloop, then $\mathrm{\# F}_{G}(k)=0$.

(3) If $e \in E$ is a loop, then $\# \mathrm{~F}_{G}(k)=(k+1) \cdot \# \mathrm{~F}_{G \backslash e}(k)$.

(4) If $e \in E$ is neither a loop nor a coloop, then $\# \mathrm{~F}_{G}(k)=\# \mathrm{~F}_{G \backslash e}(k)+\# \mathrm{~F}_{G / e}(k)$. 
To show this theorem we examine how a $\mathbb{Z}_{k}$-flow on $G$ induces $\mathbb{Z}_{k}$-flows on $G_{/ e}$ and $G_{\backslash e}$, respectively, and how a totally cyclic reorientation of $G$ induces totally cyclic reorientations of $G_{/ e}$ and $G_{\backslash e}$, respectively. We first turn our attention to the $\mathbb{Z}_{k}$-flows.

Lemma A.2 Let $G=(V, E)$ be an oriented graph and $e \in E$ neither a loop nor a coloop. If $f$ is a $\mathbb{Z}_{k}$-flow on $G$, then $\left.f\right|_{E \backslash e}$ is

(1) $a \mathbb{Z}_{k}$-flow on $G_{/ e}$ and

(2) $a \mathbb{Z}_{k}$-flow on $G_{\backslash e}$ if and only if $f(e)=0$.

Proof Let $e=u v$. At any vertex $w \notin\{u, v\}$ the flow $(A f)_{v}$ does not change when passing from $G$ to $G_{/ e}$ or $G_{\backslash e}$. In $G_{/ e}$ the vertices $u$ and $v$ have been identified to form a vertex $u^{\prime}$ and $\left(\left.A_{G_{l e}} f\right|_{E \backslash e}\right)_{u^{\prime}}=(A f)_{u}+(A f)_{v}=0$. In $G_{\backslash e}$ we have $\left(\left.A_{G_{\backslash e}} f\right|_{E \backslash e}\right)_{u^{\prime}}=(A f)_{u}-f(e)$ which is zero if and only if $f(e)=0$, and similarly for $v$.

So a $\mathbb{Z}_{k}$-flow on $G$ induces a $\mathbb{Z}_{k}$-flow on $G_{/ e}$ and if $f(e)=0$ it also induces a $\mathbb{Z}_{k}$-flow on $G_{\backslash e}$. Moreover it turns out that any $\mathbb{Z}_{k}$-flow on $G_{/ e}$ is induced by a unique $\mathbb{Z}_{k}$-flow on $G$ and the same holds for $G \backslash e$.

Lemma A.3 Let $G=(V, E)$ be an oriented graph and $e \in E$ neither a loop nor a coloop.

(1) Given a $\mathbb{Z}_{k}$-flow $f^{\prime}$ on $G_{/ e}$ there is a unique $\mathbb{Z}_{k}$-flow $f$ on $G$ such that $\left.f\right|_{E \backslash e}=f^{\prime}$.

(2) Given a $\mathbb{Z}_{k}$-flow $f^{\prime}$ on $G_{\backslash e}$ there is a unique $\mathbb{Z}_{k}$-flow $f$ on $G$ such that $\left.f\right|_{E \backslash e}=f^{\prime}$. Moreover this flow has the property $f(e)=0$.

Proof In both cases, we necessarily have $f\left(e^{\prime}\right)=f^{\prime}\left(e^{\prime}\right)$ for all $e^{\prime} \neq e$ and we have to check that there is unique choice for $f(e)$ that makes $f$ a $\mathbb{Z}_{k}$-flow. Let $e=u v$ oriented from $u$ to $v$. Let $A^{*}$ denote the incidence matrix of $G$ with the column corresponding to $e$ removed. In both cases $(A f)_{u}=\left(A^{*} f\right)_{u}-f(e)$ and $(A f)_{v}=\left(A^{*} f\right)_{v}+f(e)$. So $f$ is a $\mathbb{Z}_{k}$-flow if and only if $f(e)=\left(A^{*} f\right)_{u}$ and $f(e)=-\left(A^{*} f\right)_{v}$. In the first case these two values coincide because $\left(A^{*} f\right)_{u}+\left(A^{*} f\right)_{v}=\left(A_{G_{/ e}} f^{\prime}\right)_{u^{\prime}}=0$ where $u^{\prime}$ is the vertex obtained by identifying $u$ and $v$. In the second case, both of these values are zero, because $\left(A^{*} f\right)_{u}=\left(A_{G_{\backslash e}} f^{\prime}\right)_{u}=0$ and $\left(A^{*} f\right)_{v}=\left(A_{G_{\backslash e}} f^{\prime}\right)_{v}=0$.

Now we turn to totally cyclic reorientations. Here the situation is a bit more complicated compared to $\mathbb{Z}_{k}$-flows. We start with a useful characterization of totally cyclic orientations.

Lemma A.4 Let $G$ be an oriented graph. $\sigma$ is a totally cyclic reorientation of $G$ if and only iffor any vertices $u, v \in V$ in the same component of the underlying undirected graph, there exists a directed path in $\sigma$ G from $u$ to $v$.

Proof Suppose $\sigma$ is totally cyclic. As both $u$ and $v$ lie in the same component of the undirected graph, there is an undirected path $P$ from $u$ to $v$. As ${ }_{\sigma} G$ is totally cyclic, every edge of $P$ lies on a directed cycle. In a directed cycle, there is a directed path from any vertex to any other vertex. So for any edge $u_{i} v_{i}$ in $P$ there is a directed path in $G$ from $u_{i}$ to $v_{i}$. Concatenating all these paths, we obtain a directed walk in $G$ from $u$ to $v$, which in particular contains a directed path from $u$ to $v$ as a subgraph.

Conversely, suppose we can always find a directed path from any vertex to any other. Let $e$ be an edge oriented from $u$ to $v$. Then the assumption guarantees the existence of a path $P$ from $v$ to $u$. Concatenating $P$ and $e$ yields a directed cycle.

In the following we use $\Delta$ to denote the symmetric difference of sets. So given a reorientation $\sigma$ and an edge $e, \sigma \Delta e$ is the reorientation obtained from $\sigma$ by reversing the edge $e$. 
Lemma A.5 Let $G=(V, E)$ be an oriented graph and $e \in E$ neither a loop nor a coloop. Let $\sigma$ be a totally cyclic reorientation of $G$. Then

(1) $\sigma \cap(E \backslash e)$ is a totally cyclic reorientation of $G_{/ e}$, and

(2) $\sigma \cap(E \backslash e)$ is a totally cyclic reorientation of $G_{\backslash e}$ if and only if both $\sigma$ and $\sigma \Delta e$ are totally cyclic reorientations of $G$.

Proof (1) As ${ }_{\sigma} G$ is totally cyclic, there is a collection $\mathcal{C}$ of directed cycles in ${ }_{\sigma} G$ that cover all edges. Then $\left\{C_{/ e} \mid C \in \mathcal{C}\right\}$ is a collection of directed cycles in $\sigma \cap(E \backslash e) G_{/ e}$ that covers all edges in $G_{/ e}$ and hence $\sigma \cap(E \backslash e) G_{/ e}$ is totally cyclic.

(2) Let $e=u v$. Suppose $\sigma \cap(E \backslash e) G \backslash e$ is totally cyclic. Then by Lemma A.4 there exist directed paths from $u$ to $v$ and from $v$ to $u$. These show that no matter which way we orient $e$, we can always find a directed cycle on which $e$ lies and so both ${ }_{\sigma} G$ and ${ }_{\sigma \Delta e} G$ are totally cyclic.

Conversely, suppose both ${ }_{\sigma} G$ and ${ }_{\sigma \Delta e} G$ are totally cyclic. The edge $e$ lies on a directed cycle in ${ }_{\sigma \Delta e} G$ so by Lemma A.4 there is a directed path $P$ from $u$ to $v$ in ${ }_{\sigma} G_{\backslash e}$. Let $u^{\prime}, v^{\prime}$ be any two vertices in $G \backslash e$. As ${ }_{\sigma} G$ is totally cyclic there is a directed path $P^{\prime}$ in ${ }_{\sigma} G$ from $u^{\prime}$ to $v^{\prime}$. We replace every occurrence of $e$ in $P^{\prime}$ with $P$ and obtain a directed walk (and hence a

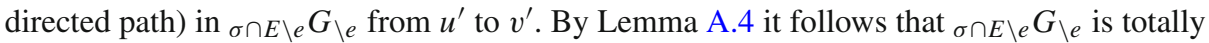
cyclic.

Lemma A.6 Let $G=(V, E)$ be an oriented graph and $e \in E$ neither a loop nor a coloop.

(1) Let $\sigma \subseteq E \backslash$ e be a totally cyclic reorientation of $G_{/ e}$. Then at least one of $\sigma$ and $\sigma \cup e$ is a totally cyclic reorientation of $G$.

(2) Let $\sigma \subseteq E \backslash e$ be a totally cyclic reorientation of $G_{\backslash e}$. Then both $\sigma$ and $\sigma \cup e$ are totally cyclic reorientations of $G$.

Proof (1) Let $\sigma G_{/ e}$ be totally cyclic and $e=u v$. Let $\mathcal{C}$ be a collection of directed cycles in $G_{/ e}$ that covers all edges of $G_{/ e}$. Now we distinguish two cases: Is one of these cycles "broken" in $G$ or not? More precisely does there exist a cycle $C \in \mathcal{C}$ that contains consecutive edges $e_{1}$ and $e_{2}$ such that $e_{1}$ enters $u$ and $e_{2}$ leaves $v$ (or vice versa)? ${ }^{1}$ If not, then $\mathcal{C}$ shows that ${ }_{\sigma} G_{\backslash e}$ is also totally cyclic and we can continue as in part (2) below. So we suppose that $C$ is such a broken cycle. In this case $C$ gives a directed path from $v$ to $u$ in $G$. We now orient $e$ from $u$ to $v$. Then any directed path $P$ in $G_{/ e}$ from a vertex $u^{\prime}$ to a vertex $v^{\prime}$ can be turned into a directed path in $G$ from $u^{\prime}$ to $v^{\prime}$ by substituting the edge $e$ or the path given by $C$ wherever $P$ is broken. Using Lemma A.4 the claim follows.

(2) Already in $G_{\backslash e}$ there is, for any two vertices $u, v$ in the same component, a directed path from $u$ to $v$. This remains true after the edge $e$ is inserted, no matter how $e$ is oriented (note that $e$ is not a coloop). So by Lemma A.4 both $\sigma$ and $\sigma \cup e$ are totally cyclic reorientations of $G$.

Now we have all ingredients to show that $\mathrm{HF}_{G}(k)$ is a Tutte-Grothendieck invariant.

Proof of Theorem A.1 (1) If $E=\emptyset$, then $\mathrm{F}_{G}(k)=\{(\emptyset, \emptyset)\}$.

(2) If $e \in E$ is a coloop, then any flow $f$ on $G$ has $f(e)=0$. Thus $e$ is also a coloop in $G / \operatorname{supp}(f)$ which means that there is no totally cyclic orientation on $G / \operatorname{supp}(f)$. So $\mathrm{F}_{G}(k)=\emptyset$.

1 We also require that $C$ does not consist of a single edge that is a loop. 
(3) If $e \in E$ is a loop, then $(f, \sigma) \mapsto\left(\left.f\right|_{E \backslash e}, \sigma \cap E \backslash e\right)$ is a surjective map from $\mathrm{F}_{G}(k)$ onto $\mathrm{F}_{G \backslash e}$ and every fiber of this map has cardinality $k+1$. The reason is that given $\left(\left.f\right|_{E \backslash e}, \sigma \cap E \backslash e\right)$ we can define $f(e) \in \mathbb{Z}_{k}$ arbitrarily and $f$ will become a $\mathbb{Z}_{k}$-flow on $G$. The case $f(e)=0$ is counted twice as either orientation of $e$ will turn $\sigma$ into a totally cyclic orientation of $G / \operatorname{supp}(f)$.

(4) Let $e \in E$ be neither a coloop nor a loop. Consider the map $\pi_{G / e}: \mathrm{F}_{G}(k) \rightarrow \mathrm{F}_{G / e}(k)$ given by $(f, \sigma) \mapsto\left(\left.f\right|_{E \backslash e}, \sigma \cap E \backslash e\right)$. Lemmas A.2 and A.5 tell us that $\pi_{G / e}$ is welldefined and Lemmas A.3 and A.5 tell us that every $\left(f^{\prime}, \sigma^{\prime}\right) \in \mathrm{F}_{G / e}(k)$ has either one or two pre-images under $\pi_{G / e} .\left(f^{\prime}, \sigma^{\prime}\right)$ has two pre-images if and only if the unique $\mathbb{Z}_{k}$-flow $f$ with $\left.f\right|_{E \backslash e}=f^{\prime}$ has $f(e)=0$ and both $\sigma^{\prime}$ and $\sigma^{\prime} \cup e$ are totally cyclic reorientations of $G_{/ \operatorname{supp}(f)}$.

Loosely speaking, this means that the cardinalities of $\mathrm{F}_{G}(k)$ and $\mathrm{F}_{G / e}(k)$ are the same, except that we have to count those $\left(f^{\prime}, \sigma^{\prime}\right) \in \mathrm{F}_{G / e}(k)$ that have two pre-images twice. So let $\mathrm{F}_{G}^{\prime}(k)$ denote the set of all $(f, \sigma) \in \mathrm{F}_{G}(k)$ such that $f(e)=0$ and both $\sigma$ and $\sigma \Delta e$ are totally cyclic reorientations on $G / \operatorname{supp} f$. Consider the map $\pi_{G \backslash e}: \mathrm{F}_{G}^{\prime}(k) \rightarrow \mathrm{F}_{G \backslash e}(k)$ given by $(f, \sigma) \mapsto\left(\left.f\right|_{E \backslash e}, \sigma \cap E \backslash e\right)$. Lemmas A.2 and A.5 tell us that $\pi_{G \backslash e}$ is welldefined and Lemmas A.3 and A.5 tell us that every $\left(f^{\prime}, \sigma^{\prime}\right) \in F_{G \backslash e}(k)$ has precisely two pre-images under $\pi_{G \backslash e}$. But this means that $\#_{G}(k)=\#_{G / e}(k)+\# \mathrm{~F}_{G \backslash e}(k)$ as desired.

\section{Appendix B: Combinatorial proof of the Tutte interpretation}

In this section we give a combinatorial proof of Theorem 5.1, our interpretation of $t_{G}(1+\ell$, $1+k)$. The approach is similar to that in Appendix A: we show that the counting function, that we claim is identical to the Tutte polynomial, is a Tutte-Grothendieck invariant with the appropriate structure constants. Surprisingly, the combinatorial proof of Theorem 5.1 is much simpler than the combinatorial proof of Theorem 2.2.

Theorem 5.1 states that $t_{G}(1+\ell, 1+k)$ counts the number of triples $(f, t, \sigma)$ where $f$ and $t$ are, respectively, a $\mathbb{Z}_{k}$-flow and a $\mathbb{Z}_{l}$-tension on $G$ with disjoint support and $\sigma \subseteq$ $E \backslash \operatorname{supp}(f) \cup \operatorname{supp}(t)$. Now, for any edge set $S \subseteq E$, the $\mathbb{Z}_{k}$-flows $f$ on $G$ with $f(e)=0$ for all $e \in S$ are in bijection with the $\mathbb{Z}_{k}$-flows on $G_{\backslash S}$. Correspondingly, for any edge set $S \subseteq E$, the $\mathbb{Z}_{l}$-tensions $t$ on $G$ with $t(e)=0$ for all $e \in S$ are in bijection with the $\mathbb{Z}_{l}$-tensions on $G / S$. So if we define the sets $\mathrm{T}_{G}(\ell, k)$ by

$$
\begin{aligned}
\mathrm{T}_{G}(\ell, k)= & \{(S, t, f): S \subseteq E, \\
& t \text { a } \mathbb{Z}_{\ell} \text {-tension on } G / S, \\
& \left.f \text { a } \mathbb{Z}_{k} \text {-flow on } G[S]\right\},
\end{aligned}
$$

for $k, \ell \in \mathbb{N}$, Theorem 5.1 then becomes $t_{G}(1+\ell, 1+k)=\# \mathrm{~T}_{G}(\ell, k)$ for all $\ell, k \geq 1$. By the fact that the Tutte polynomial is a Tutte-Grothendieck invariant, all we have to show is the following:

(1) If $E=\emptyset$, then $\# \mathrm{~T}_{G}(\ell, k)=1$.

(2) If $e \in E$ is a coloop, then $\# \mathrm{~T}_{G}(\ell, k)=(1+\ell) \cdot \# \mathrm{~T}_{G / e}(\ell, k)$.

(3) If $e \in E$ is a loop, then $\# \mathrm{~T}_{G}(\ell, k)=(1+k) \cdot \# \mathrm{~T}_{G \backslash e}(\ell, k)$.

(4) If $e \in E$ is neither a loop nor a coloop, then $\# \mathrm{~T}_{G}(\ell, k)=\# \mathrm{~T}_{G / e}(\ell, k)+\# \mathrm{~T}_{G \backslash e}(\ell, k)$.

For any statement $A$ we will denote by $[A]$ the number 1 if $A$ holds and 0 if $A$ does not hold. Using this shorthand notation and the fact that if $e$ is a loop or a coloop then $t_{G_{\backslash e}}=t_{G_{/ e}}$, we 
can write what we have to show more compactly as

$$
\# \mathrm{~T}_{G}(\ell, k)=\ell^{[e \text { is a coloop }]} \# \mathrm{~T}_{G \backslash e}(\ell, k)+k^{[e \text { is a loop }]} \# \mathrm{~T}_{G / e}(\ell, k) .
$$

Before we show that this identity holds, we work out how the $\mathbb{Z}_{l}$-tensions on $G$ and on $G \backslash e$ are related, just as we did in Appendix A for $\mathbb{Z}_{k}$-flows.

Given a map $f: E \rightarrow \mathbb{Z}_{k}$ and a set $S \subseteq E$ we define $\left.f\right|_{G_{/ S}}$ and $\left.f\right|_{G_{\backslash S}}$ to be the maps obtained by restricting $f$ to the respective edge sets of $G_{/ S}$ and $G \backslash S$. A fiber of a map $f$ is the set $f^{-1}(x)$ for any $x$ in the image.

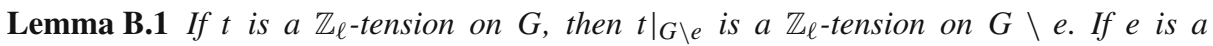
coloop, every fiber of the map $\left.t \mapsto t\right|_{G \backslash e}$ has cardinality $k$. Otherwise every fiber of the map $\left.t \mapsto t\right|_{G \backslash e}$ has cardinality 1 .

Proof A cycle in $G \backslash e$ is also a cycle in $G$. If $\langle C, t\rangle=0$ holds for every cycle $C$ of $G$, then it also holds for every cycle of $G_{\backslash e}$. So $\left.t\right|_{G_{\backslash e}}$ is a $\mathbb{Z}_{k}$-tension on $G_{\backslash e}$.

Now suppose $e$ is not a coloop in $G$. Let $t^{\prime}$ be a $\mathbb{Z}_{k}$-tension on $G_{\backslash e}$. Which $\mathbb{Z}_{\ell}$-tensions $t$ on $G$ have $\left.t\right|_{G \backslash e}=t^{\prime}$ ? Necessarily, $t\left(e^{\prime}\right):=t^{\prime}\left(e^{\prime}\right)$ for all $e^{\prime} \neq e$. All we have to show is that there is a unique choice of $t(e)$ such that $t$ is a tension. Now as $e$ is not a coloop, $e$ lies on a cycle $C$. The weights of all other edges on $C$ are fixed. As $\mathbb{Z}_{k}$ is a group, there is a unique choice of $t(e)$ such that $\langle C, t\rangle=0 . t(e)$ does not depend on the choice of $C$, as $\left\langle C^{\prime}, t\right\rangle=\left\langle C^{\prime}, t^{\prime}\right\rangle=0$ for all cycles $C^{\prime}$ that do not contain $e$.

If $e$ is a coloop, then $e$ does not lie on any cycle and so we can choose $t(e) \in \mathbb{Z}_{k}$ arbitrarily.

Now the proof of our interpretation of the Tutte polynomial is easy.

Proof of Theorem 5.1 We have to show that (2) holds. To that end we define a map

$$
\begin{aligned}
\mathrm{T}_{G}(\ell, k) & \rightarrow \mathrm{T}_{G \backslash e}(\ell, k) \uplus \mathrm{T}_{G / e}(\ell, k) \\
(S, t, f) & \mapsto\left\{\begin{array}{cc}
\left(S,\left.t\right|_{G \backslash e}, f\right) & \text { if } e \notin S, \\
\left(S \backslash e, t,\left.f\right|_{G / e}\right) & \text { if } e \in S .
\end{array}\right.
\end{aligned}
$$

By Lemma B.1 a fiber over $\mathrm{T}_{G \backslash e}(\ell, k)$ has cardinality $\ell$ if $e$ is a coloop and cardinality 1 otherwise. As we have seen in Appendix A, a fiber over $\mathrm{T}_{G / e}(\ell, k)$ has cardinality $k$ if $e$ is a loop and cardinality 1 otherwise. Thus

$$
\# \mathrm{~T}_{G}(\ell, k)=\ell^{[e \text { is a coloop }]} \# \mathrm{~T}_{G \backslash e}(\ell, k)+k^{[e \text { is a loop }]} \# \mathrm{~T}_{G / e}(\ell, k)
$$

for any $e \in E$.

\section{References}

1. Babson, E., Beck, M.: Minimal-distance chromatic and modular flow polynomials. (In preparation.)

2. Beck, M., Robins, S.: Computing the continuous discretely: integer-point enumeration in polyhedra. In: Undergraduate Texts in Mathematics. Springer, New York (2007)

3. Beck, M., Zaslavsky, T.: Inside-out polytopes. Adv. Math. 205, 134-162 (2006)

4. Beck, M., Zaslavsky, T.: The number of nowhere-zero flows on graphs and signed graphs. J. Comb. Theory Ser. B 96, 901-918 (2006)

5. Breuer, F.: Ham sandwiches, staircases and counting polynomials. PhD thesis, Freie Universität Berlin (2009)

6. Brylawski, T., Oxley, J.: The Tutte polynomial and its applications. In: Matroid Applications. Encyclopedia Math. Appl., vol. 40, pp. 123-225. Cambridge University Press, Cambridge (1992) 
7. Chen, B.: Orientations, lattice polytopes, and group arrangements I: chromatic and tension polynomials of graphs. Ann. Comb. 13, 425-452 (2010)

8. Chen, B., Stanley, R.P.: Orientations, lattice polytopes, and group arrangements II: modular and integral flow polynomials of graphs. Preprint

9. Ehrhart, E.: Polynômes arithmétiques et méthode des polyèdres en combinatoire. International Series of Numerical Mathematics, vol. 35. Birkhäuser Verlag, Basel (1977)

10. Gioan, E.: Enumerating degree sequences in digraphs and a cycle-cocycle reversing system. Eur. J. Comb. 28, 1351-1366 (2007)

11. Greene, C., Zaslavsky, T.: On the interpretation of Whitney numbers through arrangements of hyperplanes, zonotopes, non-Radon partitions, and orientations of graphs. Trans. Am. Math. Soc. 280, 97-126 (1983)

12. Kochol, M.: Polynomials associated with nowhere-zero flows. J. Comb. Theory Ser. B 84, 260-269 (2002)

13. Kook, W., Reiner, V., Stanton, D.: A convolution formula for the Tutte polynomial. J. Comb. Theory Ser. B 76, 297-300 (1999)

14. Matoušek, J.: Lectures on discrete geometry. In: Graduate Texts in Mathematics, vol. 212. Springer-Verlag, New York (2002)

15. Reiner, V.: An interpretation for the Tutte polynomial. Eur. J. Comb. 20, 149-161 (1999)

16. Schrijver, A.: Theory of linear and integer programming. In: Wiley-Interscience Series in Discrete Mathematics. Wiley, Chichester (1986)

17. Schrijver, A.: Combinatorial optimization. Polyhedra and efficiency. Volume A. In: Algorithms and Combinatorics, vol. 24. Springer, Berlin (2003)

18. Stanley, R.P.: Acyclic orientations of graphs. Discrete Math. 5, 171-178 (1973)

19. Stanley, R.P.: Combinatorial reciprocity theorems. Adv. Math. 14, 194-253 (1974)

20. Tutte, W.T.: A ring in graph theory. Proc. Camb. Philos. Soc. 43, 26-40 (1947)

21. Tutte, W.T.: A contribution to the theory of chromatic polynomials. Can. J. Math. 6, 80-91 (1954) 